\title{
Metastatic gastric cancer of the testis diagnosed through urine cytology
}

\author{
Mee Sook Roh, Song-Hee Han \\ Department of Pathology, Dong-A University College of Medicine, Busan, Korea
}

Gastric carcinoma (GC) is the most common malignancy in Korea [1]. The common metastatic sites of GC include the regional lymph nodes, liver, peritoneum, bone marrow, and lung. Metastasis of GC to the male genital tract, including the testis, epididymis, scrotum, and spermatic cord, is exceptionally unusual in clinical practice [2]. Although previous studies have reported fewer than 30 cases in the past 70 years, detection of metastatic gastric adenocarcinoma in urine cytology is unique [2].

GC has varied histologic features, which causes problems with diagnosis through urine cytology. Our case emphasizes the importance of considering secondary lesions involving the testis in the urine cytology of relatively young patients. Although a clinical history of GC is a clue to diagnosis, this clinical information is not always available.

\section{CASE REPORT}

A 44-year-old male was admitted to our hospital with a history of swelling and pain in the left scrotum for 3 months. He was diagnosed with gastric tubular adenocarcinoma, moderately differentiated at another hospital in July 2016 (Fig. 1). He underwent radical subtotal gastrectomy for GC at another hospital and was classified as American Joint Committee on Cancer pathologic stage pT3N3. One year later, the patient received postoperative adjuvant chemotherapy with FOLFOX (oxaliplatin plus fluorouracil and leucovorin) for 14 cycles. The ensuing computed tomography scan suggested massive peritoneal seeding. Staging laparoscopic examination revealed intraperitoneal dis-

Received: March 30, 2021 Revised: April 16, 2021 Accepted: April 19, 2021

Corresponding Author: Song-Hee Han, MD

Department of Pathology, Dong-A University College of Medicine, 32 Daesingongwon-ro, Seo-gu, Busan 49201, Korea

Tel: +82-51-240-2863, Fax: +82-51-243-7396, E-mail: pshsh625@gmail.com seminated nodules in the transverse and sigmoid colon and the liver. The patient received 21 courses of intraperitoneal chemotherapy for 20 months. Five months later, the patient presented with progressive scrotal pain and swelling. Scrotal ultrasonography revealed an ill-defined hypoechoic lesion and a large fluid collection in the left scrotal sac. His serum levels of lactate dehydrogenase (LDH), $\alpha$-fetoprotein (AFP), and $\beta$-human chorionic gonadotropin ( $\beta$-HCG) were normal. Routine voided urine cytology (ThinPrep, Hologic, Inc., Marlborough, MA, USA) revealed numerous, overlapping, 2-dimensional sheets with central lumina formation (Fig. 2A). The tumor cells showed vacuolated cytoplasm with moderate nuclear pleomorphism, coarse chromatin, and prominent nucleoli (Fig. 2B). Radical orchiectomy was performed at the same time. On gross examination, the testis was replaced partly by an infiltrating whitish mass measuring $3.4 \times 1.6$ $\mathrm{cm}$ (Fig. 1C). Histologically, the tumor showed irregularly distended or fused tubular or glandular structures in a background of atrophic testis (Fig. 1D). The cells exhibited variable amounts of intraluminal mucin with nuclear pleomorphism. Abundant lymphatic and vascular invasions were observed. Based on the pathologic features of the patient's previous original hematoxylin and eosin slide and his clinical history, a diagnosis of metastatic adenocarcinoma from urine cytology and surgical specimen was established. Although the patient subsequently received chemoradiotherapy, he died of complications two months later.

\section{DISCUSSION}

In cases with a clinical history of gastric cancer, diagnosis of metastasis based on cytology is uncomplicated and can be confirmed through review of hematoxylin and eosin-stained slides and immunohistochemistry. For cases that lack clinical history of a primary lesion, however, the differential diagnosis is difficult and supported by few features, especially in urine cytology. 
The first consideration for differential diagnosis of abnormal cells on urine cytology is primary urinary bladder cancer, including high-grade urothelial carcinoma (HGUC) and adenocarcino- ma [3,4]. The differential diagnosis should include non-urothelial neoplasms, such as metastases from other lesions and primary testis neoplasms either by direct invasion or lymphohematoge-
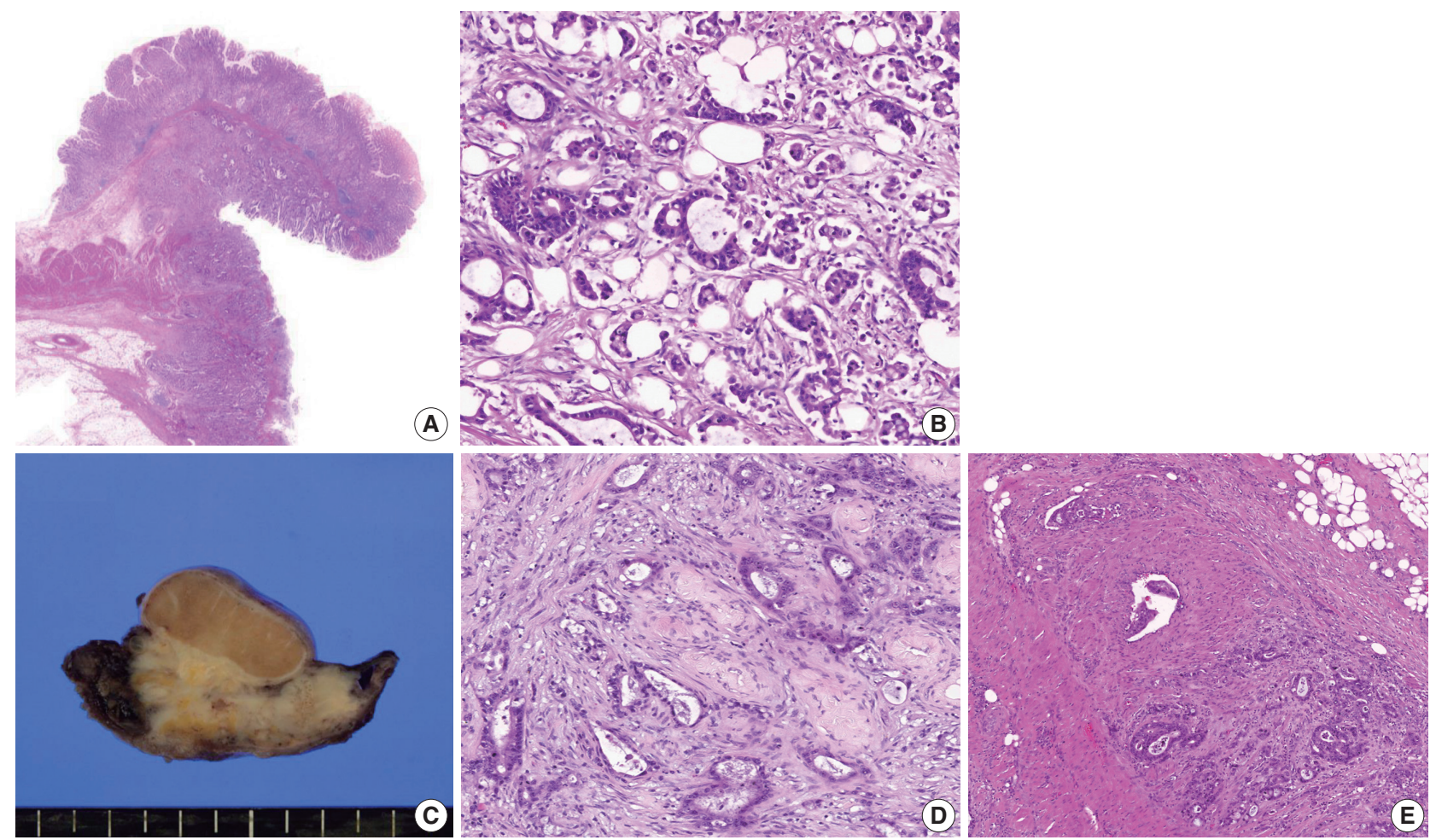

Fig. 1. Representative image of hematoxylin and eosin staining of a specimen from subtotal gastrectomy (A, B). In a radical orchiectomy specimen, an infiltrating whitish mass shows involvement of the testis $(\mathrm{C})$. Histologically, this tumor is composed of an atypical glandular structure (D) that is frequently found in lymphovascular spaces (E), compatible with metastatic adenocarcinoma.

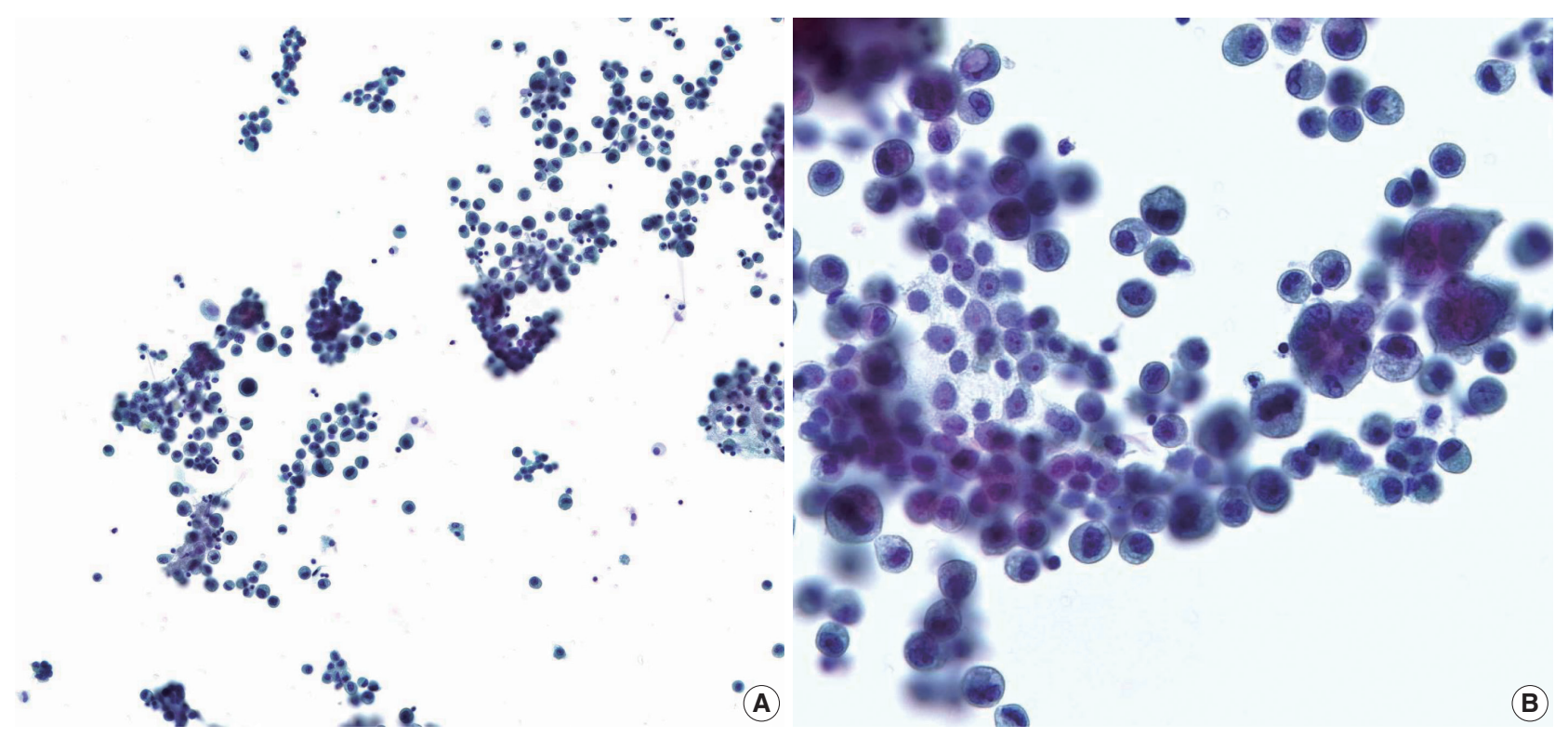

Fig. 2. Representative image of urine cytology on liquid-based preparation. (A) Voided urine cytology reveals numerous, crowded, overlapped clusters with occasional luminal formations. (B) Tumor cells show granular and vacuolated cytoplasm with moderate nuclear pleomorphism. 
nous dissemination $[3,4]$. Relatively abundant and vacuolated cytoplasm is important in the differential diagnosis of HGUC. The absence of large uniform cells with dis-cohesion mixed with lymphocytes helps distinguish seminoma, the most common neoplasm of the testis [4]. However, metastatic adenocarcinoma and primary adenocarcinoma of the urinary bladder might be indistinguishable because they are fairly similar to their counterparts in organs such as the stomach, colon, and prostate [3,5]. When encountered with this diagnostic challenge, physicians can use certain clinical and morphological characteristics to differentiate metastatic lesions. The average age at diagnosis of metastasis to the testis is older than 50 years (range, 23 to 72 years) [2]. In contrast, patients with primary testicular tumors are rarely older than 40 years [6], and those with HGUC typically are older than 70 years [7]. Tumor markers in the serum also contribute to the differential diagnosis. Carbohydrate antigen 19-9 [8,9] and carcinoembryonic antigen [9] levels, related to GC, along with AFP, $\mathrm{LDH}$, and $\beta$-HCG levels, related to primary tumors of the testis, are different according to clinical settings. Furthermore, metastatic symptoms include a palpable mass in the groin with or without pain, scrotal swelling, and hydrocele [2]. A review of the patient's clinical manifestations and histologic features can suggest metastatic lesions.

Progressive GC metastasis to the testis is extremely rare, has a poor prognosis, and is a diagnostic challenge [2]. For cases that include the possibility of metastasis, urine cytology can be a helpful screening method when correlated with clinical history of secondary lesions, especially in the testis.

\section{Ethics Statement}

This study was approved by Dong-A University Hospital Institutional Review Boards (DAUHIRB-TEMP-21-059) and informed consent was waived.

\section{Availability of Data and Material}

The datasets generated or analyzed during the study are available from the corresponding author on reasonable request.

\section{ORCID}

Mee Sook Roh https://orcid.org/0000-0002-5676-5569

Song-Hee Han https://orcid.org/0000-0002-4564-7014

\section{Author Contributions}

Conceptualization: MSR, SHH. Data curation: MSR, SHH. Funding acquisition: SHH. Investigation: MSR, SHH. Writing—original draft: SHH. Writing—review \& editing: MSR. Approval of final manuscript: all authors.

\section{Conflicts of Interest}

The authors declare that they have no potential conflicts of interest.

\section{Funding Statement}

No funding to declare.

\section{References}

1. Hong S, Won YJ, Park YR, et al. Cancer statistics in Korea: incidence, mortality, survival, and prevalence in 2017. Cancer Res Treat 2020; 52: 335-50.

2. Dai W, Liu D, Zuo J, et al. Metastatic tumor of male genital system from gastric cancer: a case report and review of literature. Int J Clin Exp Pathol 2017; 10: 8592-8.

3. Bardales RH, Pitman MB, Stanley MW, Korourian S, Suhrland MJ. Urine cytology of primary and secondary urinary bladder adenocarcinoma. Cancer 1998; 84: 335-43.

4. Alsharif M, Aslan DL, Jessurun J, Gulbahce HE, Pambuccian SE. Cytologic diagnosis of metastatic seminoma to the prostate and urinary bladder: a case report. Diagn Cytopathol 2008; 36: 734-8.

5. Tian Y, Yao W, Yang L, Wang J, Wazir R, Wang K. Primary adenocarcinoma of the rete testis: a case report and review of the literature. Oncol Lett 2014; 7: 455-7.

6. Li B, Cai H, Kang ZC, Wu H, Hou JG, Ma LY. Testicular metastasis from gastric carcinoma: a case report. World J Gastroenterol 2015; 21: 6764-8.

7. Hassan M, Solanki S, Kassouf W, et al. Impact of implementing the Paris system for reporting urine cytology in the performance of urine cytology: a correlative study of 124 cases. Am J Clin Pathol 2016; 146: 384-90.

8. Kato K, Taniguchi M, Kawakami T, et al. Gastric cancer with a very high serum CA 19-9 level. Case Rep Gastroenterol 2011; 5: 258-61.

9. Cainap C, Nagy V, Gherman A, et al. Classic tumor markers in gastric cancer: current standards and limitations. Clujul Med 2015; 88: 111-5.

\section{Code Availability}

Not applicable. 